\title{
NEW SIMULATION CODE FOR SYNCHROTRON RADIATION BASED ON A REAL BEAM ORBIT
}

\author{
Tetsuo Abe*, \\ High Energy Accelerator Research Organization (KEK), 1-1 Oho, Tsukuba, Ibaraki 305-0801, Japan
}

\begin{abstract}
A computer code to simulate synchrotron-radiation power and spatial distributions has been developed based on the method by Abe and Yamamoto [1], where a real beam orbit is obtained by fitting measurements of beamposition monitors (BPMs) with some offset corrections for BPMs and quadrupole-magnet alignments. In this paper, the status and basic performance are presented. This code has been rewritten in Fortran2003 so as to obtain expectable maximal speed-up by parallel computing, aiming at online alarm systems to take precautions against synchrotron-radiation damage, toward higher beam current accelerators.
\end{abstract}

\section{INTRODUCTION}

Synchrotron radiation (SR) is useful for beam monitoring / diagnostics in accelerator science, and for experimental studies in material science. However, it sometimes causes serious damage to accelerator or detector components of such as a high luminosity factory machine. In the previous work [1], a simulation method to protect the detector against the SR was developed, where the method is based on a real beam orbit obtained by fitting measurements of beam-position monitors (BPMs) with some offset corrections for BPMs and quadrupole-magnet alignments. The validity of the method was demonstrated for the gain-drop accident of Belle Silicon Vertex Detector [2]. Based on this method, an old version of a SR alarm software was written in Fortran77. However, the turnaround time would increase proportional to the number of magnets. Since there is almost no room for improvement on CPU clock frequencies, we need to introduce parallel computing in order to make the online software useful. For this purpose, the old code has been completely rewritten in Fortran2003 in an object-oriented form, which is adequate to parallel computing.

\section{PROGRAM COMPONENTS}

The following modules have been newly written, and combined to form an online software with a graphical user interface (GUI).

Figure 1 shows the flowchart for the new code.

\footnotetext{
*tetsuo.abe@kek.jp
}

05 Beam Dynamics and Electromagnetic Fields

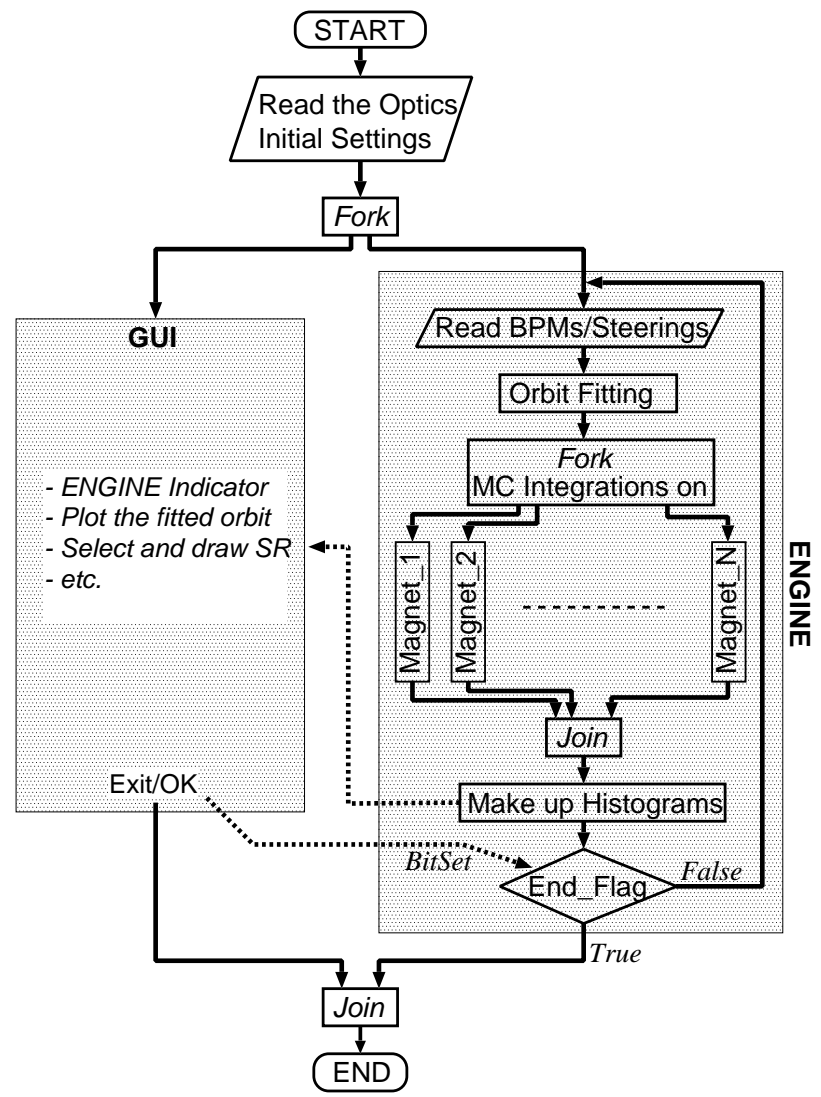

Figure 1: Flowchart for the new code. Dotted arrows indicate data transfer between the two main threads.

\section{Numerical Integration Module}

Since the dimension of integration to obtain SR power and spatial distributions is seven at maximum, including a simulation of a beam size, a Monte Carlo (MC) method is appropriate. In this module, the iterative importancesampling method is employed as in VEGAS [3] and BASES [4]. The data structure is designed to have an array containing one thousand different functions at maximum, each of which has a distributed-memory in parallel computing. This module possesses one- or two-dimensional histogramming and a function to generate unweighted events of SR photons for subsequent analyses, e.g. detector simulations.

\section{Function Minimization Module}

Beam-orbit fitting is performed by minimizing a $\chi^{2}$ function of a beam orbit and BPM measurements, using a quasi-Newton method with a variable metric, where

D05 Code Developments and Simulation Techniques 


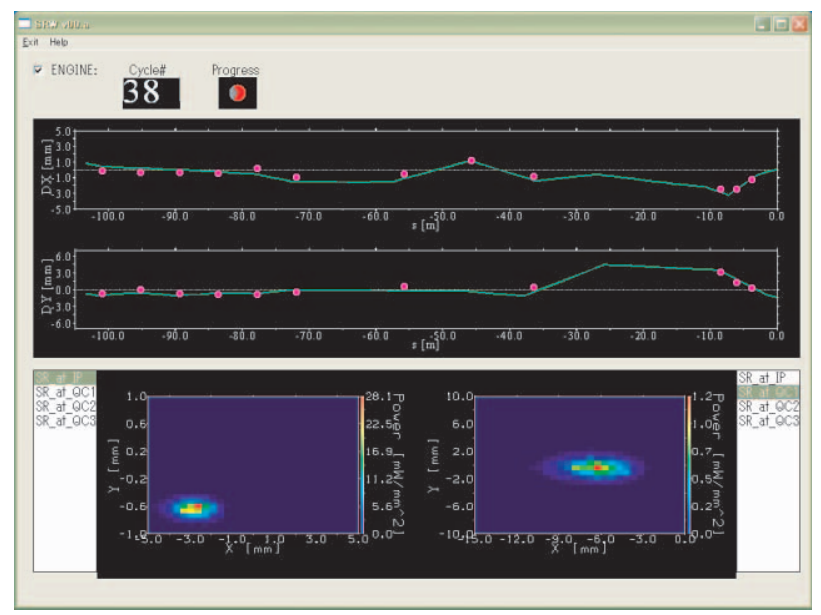

Figure 2: GUI (tentative version). Green lines indicate a fitted orbit for BPM measurements (magenta circles). Two SR spatial distributions are shown as examples. Only temporary offset corrections are applied here.

two matrix-updating formulae (DFP and BFGS) are used [5]. This method is basically the same as implemented in MINUIT [6] / MIGRAD routine, except for the special treatment when a Hessian matrix is not positive definite. Another difference is that an argument of the function to be minimized can be any user-defined structure (derived-type) because many and various types of parameters are needed for beam orbit calculations.

\section{Parallelism}

The code has been complied using gfortran from GNU Compiler Collection (GCC) [7] version 4.3. Since its version 4.2, GCC has officially supported OpenMP [8], an application program interface for multi-platform sharedmemory parallel programming in $\mathrm{C} / \mathrm{C}++$ and Fortran on all architectures. The parallel computing of the new code is performed by OpenMP implemented in gfortran.

\section{GUI}

For user inputs and graphical outputs, DISLIN [9] version 9.1 is used, which is a high-level plotting library for displaying data as curves, pie charts, contours, maps, etc, including a large variety of parameter setting routines called to create individually customized graphics. This software is available for not only several C, but also Fortran $77 / 90 / 95$ compilers, so that the interface to DISLIN is written also in Fortran.

Figure 2 is an example of snapshots of the GUI, showing a fitted orbit and SR spatial distributions.

\section{PERFORMANCE}

The first version of the new code has been just completed. The turnaround times were measured for the $100 \mathrm{~m}$ straight section of the KEKB [10] / HER upstream the interaction point, using a personal computer with an Intel Core Duo processor $(2 \mathrm{GHz})$. The typical average 05 Beam Dynamics and Electromagnetic Fields turnaround time for this example is 14.2 seconds for "OMP parallel num_threads(1)", and 9.2 seconds for "OMP parallel num_threads (2)", excluding the time of orbit fitting, where the contribution from orbit fitting to the whole turnaround time is small. Actually, this MC integration module can provide almost two times faster computations with "OMP parallel num_threads (2)" compared to "OMP parallel num_threads (1)" if the integrands are almost the same. Therefore, some scheduling algorithm using "OMP do schedule()" clause is needed to make the turnaround time minimum.

In any case, we certainly reap the benefit of parallel computing.

\section{FUTURE PROSPECTS}

The first version includes only monitoring. The most important function, which is to take precautions against SR in order to protect accelerator components or detectors, is to be implemented soon.

\section{REFERENCES}

[1] T. Abe and H. Yamamoto, Phys. Rev. ST Accel. Beams 7, 072802 (2004) [arXiv:physics/0404043].

[2] G. Alimonti et al. [BELLE Collaboration], Nucl. Instrum. Meth. A 453, 71 (2000).

[3] G. P. Lepage, J. Comput. Phys. 27, 192 (1978).

[4] S. Kawabata, Comput. Phys. Commun. 88, 309 (1995).

[5] R. Fletcher, Comput. J. 13, 317 (1970).

[6] F. James and M. Roos, Comput. Phys. Commun. 10, 343 (1975).

[7] http://gcc.gnu.org/

[8] http://www.openmp.org/drupal/

[9] http://www.mps.mpg.de/dislin/

[10] S. Kurokawa, Nucl. Instrum. Meth. A 499, 1 (2003).

D05 Code Developments and Simulation Techniques 